\title{
BXQ-350 Nanovesicle Formulation
}

National Cancer Institute

\section{Source}

National Cancer Institute. BXQ-350 Nanovesicle Formulation. NCI Thesaurus. Code C131491.

A stable, nanovesicle formulation composed of a synthetic form of the human glycoprotein saposin C (SapC) linked to the phospholipid dioleoylphosphatidylserine (DOPS), with potential antineoplastic activity. Upon intravenous administration, the BXQ350 nanovesicle formulation selectively targ ets and preferentially accumulates in tumor vessels and cells, due to the leaky nature of tumor vasculature and the presence of phosphatidylserine (PS) lipids in tumor cell membranes. Upon binding to the phospholipids in the tumor cell membrane, SapC fuses with the membrane and is internalized leading to its accumulation within the internal membrane. SapC becomes active in the acidic tumor microenvironment and as a lysosomal sphingolipid activator protein, activates lysosomal enzymes, such as beta-glucosidase, acid sphingomyelinase, and betagalactosylceramidase. This leads to the degradation of glucosylceramide and sphing omyelin, and the conversion of galactosylceramide to ceramide, respectively. This elevates intracellular ceramide levels, activates caspases and induces ceramide-mediated apoptosis, which together lead to an inhibition of tumor cell growth. SapC plays key roles in lipid transport and organization of biological membranes and has strong lipid membrane binding activity. 